\title{
Long-term follow-up culture in state newborn screening programs
}

Timothy Hoff, PhD

\begin{abstract}
Purpose: Long-term follow-up is an increasing focus as newborn screening expands in the United States. The present study informs this issue by examining the role played by organizational culture in shaping the scope and substance of long-term follow-up in state newborn screening programs. Methods: Qualitative interviews were conducted with 38 state newborn screening programs. Results: Several key cultural norms were identified within state newborn screening programs that may undermine proactive attempts to conduct long-term follow-up. These include (a) beliefs that place direct patient care and specialist care versus a public health orientation at the center of long-term follow-up; (b) an everyday emphasis on short-term follow-up that obscures the longer-term follow-up focus; and (c) the perception that others are engaged in long-term follow-up at the state level. Conclusions: The findings support the importance of understanding state newborn screening program culture and how that culture may shape the scope and substance of long-term follow-up in a given state, regardless of the level of staff and resources made available to conduct these activities. Genet Med 2008:10(6):396-403.
\end{abstract}

Key Words: newborn screening, long-term follow-up, culture, state newborn screening programs, public health policy

Newborn screening (NBS) facilitates the early detection and prevention of disease in infants and children, reduces morbidity associated with disease, and improves quality of life and longevity for individuals afflicted with or susceptible to certain disorders. ${ }^{1}$ Currently, NBS programs exist in all 50 states, the District of Columbia, and some US territories. States screen for anywhere from 8 to 50 genetic and metabolic disorders, although with the new recommended minimum panel, ${ }^{2}$ many states have expanded screening to 30 or more disorders. ${ }^{3}$

The issue of follow-up for newborns with abnormal screens or diagnosed disorders is a particularly important issue, because NBS is screening, not diagnosis. ${ }^{4}$ Short-term follow-up (STFU) involves the state NBS program communicating abnormal test results to families, primary care providers (PCP), and specialists; initial education of families and PCPs regarding the disorder in question and possible testing outcomes; referral of patients to appropriate clinicians for additional testing; location of appropriate PCPs for patients; and gathering of confirmatory test results. ${ }^{1}$

From the Department of Health Policy and Management, School of Public Health, University at Albany, Rensselaer, New York.

Timothy Hoff, PhD, Department of Health Policy and Management, School of Public Health, University at Albany, SUNY, Room 181, GEC Building, 1 University Place, NY 12144. E-mail: thoff@albany.edu.

Disclosure: The author declare no conflict of interest.

A supplementry appendix is available via the ArticlePlus feature at www.geneticsinmedicine.org. Please go to the June issue and click on the ArticlePlus link posted with the article in the Table of Contents to view this material.

Submitted for publication January 24, 2008.

Accepted for publication February 15, 2008.

DOI: $10.1097 /$ GIM.0b013e3181770212
Many state NBS programs have well defined STFU protocols that accompany their testing activities. However, long-term follow-up (LTFU) is a more controversial, less understood component of the NBS system in the United States. ${ }^{5,6}$ In addition to initial screening and STFU, LTFU is the third major component of NBS that makes it a meaningful public health activity. ${ }^{7}$ A general definition has been provided by the Clinical and Laboratory Standards Institute:

"Long-term follow-up is a mechanism for determining if the newborn screening system is accomplishing its intended goal of improving health outcomes. Long-term follow-up includes an infrastructure with the capacity for periodic monitoring of selected outcome indicators appropriate for evaluating the efficacy of newborn screening. Data obtained through long-term follow-up can be useful in improving and refining the newborn screening system. Long-term follow-up may include facilitation of services to ensure coordinated, comprehensive care for the affected individual and family." 8

From a public health perspective, LTFU includes activities linked to one or more core public health functions such as quality assurance and surveillance. It may also invoke a significant role for government in the monitoring of care provided to individuals who have confirmed diagnoses for tested disorders. From the perspective of the NBS community, however, LTFU may mean a narrower set of activities which vary greatly by type of disorder and which fall under the purview of the clinical specialists and primary care doctors who care for diagnosed children. Variation in how LTFU is conceptualized may create differences in how it is viewed and acted upon within a given state or locality. 


\section{INCREASED EMPHASIS ON LTFU IN NBS}

The issue of how to view and organize LTFU is a subject of greater focus in the field of NBS. There is increased recognition that as testing expands better information is needed to determine the value of that testing in creating healthier individuals over time. ${ }^{9,10}$ Many of the recent disorders added to states' testing panels may not be well understood at the present time nor be curable. ${ }^{6}$ This makes it important to gather treatment and outcomes data longitudinally, assess the cost-benefit and appropriateness of care provided for certain types of disorders, and study quality of life and health system access issues associated with having a particular disorder.

Given the lack of a specific, agreed-upon definition of LTFU, and the resulting absence of standardized criteria for organizing and conducting LTFU nationally, the present study set out to better understand LTFU from the perspective of state NBS programs. State NBS programs lay at the heart of the NBS system of testing and care. First, they play the most important early role in identifying potential disease cases. Second, they are primarily responsible for getting affected newborns into care quickly to either confirm or disprove suspected diagnoses. Third, state NBS programs also have the legitimacy as government entities to pursue population-based, public health goals related to assuring quality care for diagnosed individuals longer-term.

Recent research has identified significant variation in how LTFU activities are structured within state NBS programs. ${ }^{3,4,6,11}$ It also shows that the majority of state NBS programs do little or nothing with respect to activities past the confirmatory diagnostic phase, and those that do suffer from a lack of systematic quality assurance mechanisms in place to guide activities. ${ }^{3,5,11}$ This study uses in-depth, qualitative methods to gain a deeper understanding of how state NBS programs view LTFU, and the kinds of cultural factors which shape these views.

\section{DATA AND METHODS}

This study was approved by the UAlbany Institutional Review Board, Protocol \#04-325. Interviews were conducted with NBS follow-up coordinators from 38 state NBS programs (see Table 1 for state programs participating). In addition, five individuals identified by a few states as especially knowledgeable about their follow-up practices were also interviewed. This resulted in a total interview sample size of 43 individuals. State programs that did not choose to participate in the interviews were not qualitatively different in terms of size, NBS testing requirements, or geographic location. Interviews were conducted over a 9-month time span between August 2005 and April 2006. Participants were recruited through phone and email from the state contact list maintained by the National Newborn Screening and Genetics Resource Center.

All interviews were conducted by phone. They lasted between 30 and 75 minutes, with the average interview 45 minutes. The interview protocol used is found in the Ap-
Table 1

State NBS programs participating in interview portion of study $(n=38)$

\begin{tabular}{ll}
$\begin{array}{l}\text { Reported some type of } \text { LTFU }^{a} \\
(n=21)\end{array}$ & $\begin{array}{c}\text { Report doing no LTFU } \\
(n=17)\end{array}$ \\
\hline Arkansas & New Jersey \\
Michigan & Utah \\
Oklahoma & Missouri \\
Illinois & Rhode Island \\
Iowa & Wisconsin \\
Florida & Oregon \\
North Carolina & Delaware \\
California & Alaska \\
Minnesota & New Hampshire \\
Maryland & South Carolina \\
Washington & Georgia \\
North Dakota & Kentucky \\
West Virginia & Ohio \\
Vermont & Connecticut \\
Colorado & Kansas \\
Montana & Tennessee \\
Arizona & Pennsylvania \\
\hline
\end{tabular}

Oklahoma

Hawaii

Nevada

Texas

${ }^{a}$ LTFU was defined in the study as activities conducted by a state newborn screening program after the point of confirmatory diagnosis.

pendix (available online only). Questions were open-ended and designed to stimulate discussion from participants. Probes were used with each question to ensure that appropriate topic areas were covered. State programs engaging and not engaging in LTFU were interviewed, because it was meaningful to ask the latter group their perceptions around the prospects for and realities of LTFU in their state, in addition to identifying barriers and opportunities that could affect the conduct of LTFU in their programs.

All interviews were tape recorded and transcribed into Microsoft Word. They were then incorporated as individual texts into an Atlas.ti database for coding. Atlas.ti is a popular software tool for conducting qualitative analysis. Interviews were coded using a grounded theoretical approach that involved open coding initially, followed by more selective coding that sought to aggregate open codes into more generalizable concepts and descriptive realities. ${ }^{12}$ The research team for the study worked in an iterative and collaborative fashion in coding data, jointly verifying the validity of and support for individual descriptive and analytic codes, and preparing data analysis for write up. The use of multiple researchers helps to enhance the reliability of qualitative findings. ${ }^{13}$ 


\section{RESULTS}

Approximately $45 \%$ of state programs (17/38) reported conducting no activities past the point of confirming diagnoses for children identified through NBS as potentially having a particular disorder. Thus, almost half the sample was not engaging in anything beyond the "short-term follow up" component of NBS (Table 1). Nevertheless, almost all 38 NBS programs interviewed, whether or not they reported doing (LTFU), could not articulate how precisely to conduct it at the level of their everyday operations.

Although there was general agreement in the sample among programs that did and did not conduct LTFU activities, for example, in saying that LTFU ideally means things such as regularly ensuring patients have a "medical home" or that patients are able to access the care they require for their disease, these definitions were not acted upon at the level of everyday NBS work and activities. For example, all of these programs stated during interviews that one appropriate component of LTFU was "ensuring that all patients have access to a medical home." Yet, none of the 38 state programs were assuring that all patients with disorders first identified through their screening had medical homes on an ongoing basis. This was despite universal agreement in the sample that a "medical home" consisted of one or both of the following: (a) the child's primary care physician, and (b) the treating specialist for the particular disorder (e.g., phenylketonuria, hypothyroidism). Medical home tracking was nonexistent in almost all programs, and only on select children in the few that reported doing it. Similarly, over three quarters of the 38 programs performed no information gathering on whether or not diagnosed children received timely, appropriate care.

\section{Cultural norms shaping LTFU definition and activities}

Approximately half of the 38 state programs believed that LTFU was not something within the scope of their role. With three exceptions, none of these programs were engaged in LTFU activities.

\footnotetext{
Honestly I don't see a real advantage [to doing LTFU]. I guess it sort of comes down to what you see as your role. We've got something like 1,000 individuals at this point that we've identified with the disorders we've tested for over the years, and I'm certainly not in a position to say we ought to be managing all those kids' care. Honestly I don't think individualized data would be of particular use. My personal opinion is that the state's primary role is to ensure that every child is screened and to ensure that an appropriate, immediate response is taken. I think that we do better if we don't let our scope get too out of control. We need to be aware that there are systems out there, but I don't think our prime responsibility is to create them and oversee them. (Pacific Northwest NBS Program)
}

Twenty-four of the 38 state NBS programs also believed that LTFU was something oriented more toward the direct patient care activities performed by specialists, and less the population-based approaches and activities that might occur at a governmental agency level. In this way, these programs did not agree with the larger public health definition of LTFU which included things like surveillance and quality assurance. Instead, programs viewed LTFU activities as within the purview of the clinical specialists caring for the child, i.e., metabolic centers within a state or pediatricians caring for children with hypothyroidism.

So what do you tell families and how do those kids do over time? The only way that we're going to be able to figure that out is by doing long term follow up on those kids. But I don't think it's realistic, that the state should assume responsibility to ensure that all the kids with sickle cell, for example, are getting into care. Once they've been referred to appropriate care centers let them do that. (Pacific Northwest NBS Program)

\begin{abstract}
The centers and specialists are closest to what's going on. They are in the best position to do any kind of long-term follow-up, because they are getting all the information on a regular basis, seeing the kids. We're not. We're removed from the everyday clinical world. And we [NBS programs nationally] can't even agree on what kinds of long-term data we should collect, or what we would use it for if even we agreed on which data were important. At least from the clinical level, it's the individual child and are they getting the kinds of care they need to get over time. I think it's the clinicians' job to assess it. (Midwest NBS Program)
\end{abstract}

Part of the motivation for programs defining LTFU in this way was their uniform concern that it was difficult to gain agreement on the types of population level data to collect and use for different disorders, and problematic to gain consensus on a standard set of data elements that would span across disorders.

Nobody really has a good sense of what to collect, what to look at. No one program collects data in the same way, and that's a problem. I'm not really sure what you would collect over time. I guess it depends what you want to look at. But that's been a real part of the issue. Because you can't do long-term follow-up if you don't know what you're following up. (Western NBS Program)

This belief seemed to lessen the empowerment felt by state NBS programs to conduct LTFU. Across all 38 programs, there was concern expressed that until there were national standards for which type of LTFU data to collect, LTFU would have to remain essentially an individual patient level activity, i.e., tracking individual patients through care, assessing outcomes for individuals and not groups of patients, and doing LTFU only on select patients.

Another cultural norm feeding into the collective belief that specialists were in the most appropriate position to conduct LTFU involved state NBS program staff expressing doubt that they had the knowledge or training to provide appropriate oversight or quality management in the clinical care arena, given the presence of highly focused specialty centers and providers in their state. This belief contributed to another feeling, unsubstantiated by any evidence they may have had, of implicit confidence on the part of NBS program staff that diagnosed newborns and children were adequately cared for through 
whatever clinical system was appropriate for their disease. This feeling of deference toward specialists also manifested itself in the feelings expressed by many NBS programs that the state's involvement in any type of LTFU was akin to "telling specialists what to do with their patients."

Once you get someone to the subspecialist, at this point in time at least, we're just assuming that, I mean once we get them diagnosed to a subspecialist, it's not our place to baby-sit them there. I don't presume to know more about how often a congenital hypothyroid patient should be followed. I'm no endocrinologist. (Midwest NBS Program)

We do not evaluate them, their doctor's performance and how they treat kids with disorders. We don't tell them how to manage their children. (Midwest NBS Program)

Basically I can tell you how many [children] received services, how many have their visits with the metabolic specialist or pediatric subspecialist. I'm not getting involved, but from my perspective I want to make sure that they're getting to the specialist. And if they're getting to the specialist then I'm satisfied that they're getting the services they need. (Southern NBS Program)

The treatment centers do provide us information on the kids that we refer whether they have insurance or not. But we don't actively monitor it. You know, when those clinical notes come in, we don't read them, and we don't have a physician or someone here review them and say, "oh, gee, you know, the treatment center should be doing this and not that." I mean we have a contract with them and they are the experts. (Mid-Atlantic NBS Program)

A handful of these same NBS programs also believed that LTFU had limited benefit for the NBS system as a whole, once it was verified that children were initially in care. A key dynamic shaping the beliefs of almost all of the programs believing that LTFU was not part of their role were their perceptions of a lack of staff and other resources available.

You must have the staff do these things [LTFU activities]. And in our program we've had some real staffing issues, and our state is having financial difficulties. We had a big layoff in April. You also need a person on board who has the medical expertise to know on the patient care level what's not being met and what needs to be corrected in terms of care delivery or access to care. (Midwest NBS Program)

The big thing is funding. I know that a lot of states are very limited in what they can do. I think we're fortunate in that we're funded through state appropriations, but if we're going to expand and take on more conditions, we could run into, you know, the dry well concept where we may not be able to do what we have done with the six conditions that we do now. (Mid-Atlantic NBS Program)

Staff is real limited here. About 4 or 5 years ago they had cut the child health programs. Of course, we still do newborn screening, and but it's just me and one other person doing it. That's all. So we're limited in what we can do around LTFU. (Southern NBS Program)

It's all about the resources you have. We've had to expand the number of disorders we test, with little increase in the funding to help absorb those new tests. We can't raise our fee right now, and the state won't give us any new money. What do you do with something like long-term follow-up when you barely have enough staff and funds to test the kids and make sure that abnormals are followed? (Southern NBS Program)

\section{Feelings of empowerment around LTFU}

For a majority of state NBS programs that reported no involvement in LTFU activities, there seemed to be an "out of sight, out of mind" mentality in place with respect to children who initially got screened and diagnosed through their program. For example, most NBS programs that initiated referrals from their program to another state-level program like Early Intervention (EI) or Children with Special Health care Needs (CSHN), for subsequent LTFU services, reported not working or following up with these other programs in getting ongoing information on how diagnosed children progressed or accessed care over time. Although programs like CSHN might be gathering these types of data, most NBS programs were unaware of what the data conveyed, and whether or not they could access it. These programs spoke of other child health programs as separate entities, in some cases daunting bureaucracies, which presented barriers in dealing with them. It was not clear how much of this was proven experience on their part, or perception rooted in their felt inability to do LTFU:

After we make sure of the diagnosis, then it goes over to our children with special health care needs or early intervention programs, and they follow them. I think the system then becomes a lot looser and I do not have a $100 \%$ of the details about what they do or don't do. (Mid-Atlantic NBS Program)

We still feel that the kids are being taken care of appropriately by the different specialists in the state. It's just that there's no formal way of reporting that data to us. Other programs are supposed to collect that kind of data, since it's more in their missions to do so. (Pacific Northwest NBS Program)

We can only do so much here, but we have other programs that are supposed to pick up the kids and track them. They have the money and staff to do so. (Northeast NBS Program)

For state programs reporting LTFU activity (i.e., activity past the confirmatory diagnostic phase), there was less confidence that the activities were the right or appropriate ones. This created wide variation in how similar LTFU goals were approached across programs. For example, 8 of the 21 state programs doing some type of LTFU reported not collecting any type of patient or service-related data past the confirmatory diagnostic phase. They also did not have formal systems or policies in place that would use collected data systematically. The other 13 programs doing LTFU reported collecting data past the confirmatory diagnostic phase. But there was large variation across these programs both in terms of where data came from, how detailed it was, and how it was received and stored within the particular NBS program. This variation was not disorder-specific. Rather, even for the same types of disorders state NBS programs collected different data, in different ways, and from different providers. 
We only ask the primary care doctor for information, regardless of the disorder. We ask them if the child's still under their care. We try to verify the parents' address and phone number, and we also ask them, like for sickle cell, if the child's still on the medication, the Penicillin. So it's basically are you still seeing the child, and generally how the child's been doing, and they'll make a comment. Very general. We get it all on a simple one page paper form, and we file it away here at the program, and put some of it on an Excel spreadsheet. (Midwest NBS Program)

We ask if the specialist is currently caring for the child just to get that out of the way up front. And if not, do they know who is caring for the child. We ask for the current address of the parents or guardian and then we also ask what the current treatment is, what the compliance is to the treatment, number of hospitalizations in the past 12 months. Then we have some questions about physical assessment, weight, height, head circumference, and the percentile of all those. And then finally we just ask if the physician or specialist feels that the family might benefit from a well visit from a public health nurse or contact with another family with a child that's been diagnosed with a similar disorder. The problem is, our computer database is very antiquated. I mean, we can't run reports or anything with the data. It's just sort of in there. (Midwest NBS Program)

We still use phone and written communication mostly. The physician who runs the particular metabolic clinic might send us a report on all the cases that were found. But it's up to them. For example, we have one who likes to publish or at least look at the data. So, for example he would look at the galactosemia cases and summarize things and send us information. But it's sent whenever he either wants to send it or we ask for something specifically. (Midwest NBS Program)

We keep a small Access database that essentially is a mailing list of all the families. We don't collect much clinical information. And when a child is diagnosed and we know where they're receiving treatment we then ask occasionally for information back from the primary physician or the parent, and we try and keep up to date on contact information for the purpose of keeping them informed of periodic parent meetings, or other. (Pacific Northwest NBS Program)

We want to be more sophisticated in the tracking and we're working to do that, for example for PKU we monitor their developmental assessment. For sickle cell disease it's basically the number of visits to the specialist, that they have a primary care doctor, and then for some of these clients the nurse coordinator working with our program keeps a very extensive health care diary. And the coordinator gets the information during the clinic visits, or through other contacts with the parents. (Southern NBS Program)

These differences in data collection and use fed into variation in how NBS programs conducted activities such as tracking of patients longitudinally and assessing quality of care. Programs that collected data primarily through hard copy or verbal communication with providers stated that they did not track patient care in any systematic manner, nor could they determine whether or not large numbers of patients were getting the right kinds of care for their disorder. There was also almost no feedback provided to primary care physicians and specialists caring for diagnosed individuals.

\section{Strong STFU cultures in state NBS programs}

An additional strong finding was the extent to which almost all 38 state NBS programs were unambiguous in describing the scope and substance of their STFU activities. In this way, it was clear from the interview data that NBS programs exhibited a strong culture of STFU.

We're here to test kids and get ones who need intervention identified and into care. That's our critical focus. And we have pretty set systems in place to do that stuff, it's mandated and we have strict policies and procedures. (Western NBS Program)

I would say we're excellent in following up abnormal test results. We know how to do that and it's a time sensitive thing so everyone knows it has to be done quickly. (Midwest NBS Program)

Evidence was found primarily in the stark differences between the way follow-up coordinators discussed and conceptualized their short-term duties juxtaposed against how they described either actual or potential long-term duties. Each NBS program coordinator interviewed gave clear, detailed descriptions for the kinds of activities their program engaged in when dealing with abnormal test results (i.e., STFU). They spoke with confidence about the systems they had in place for getting abnormal test results to providers, finding lost children, getting children to appropriate consultations, and getting a confirmatory diagnosis from providers. They also spoke of a range of evaluation mechanisms with which to track and assess the STFU system of care in their state. The detail in their descriptions of these mechanisms was unmistakable:

For the metabolic diseases, once the kid is confirmed, which takes place at one of our centers, when it is appropriate, which is when we have a real presumptive positive, the child goes to the metabolic center to be worked up. And that metabolic center is whatever their insurance allows, whatever their geographical proximity is and their convenience if, in fact, they have the option of any center. In our short term follow up program, we do talk to the child's physician, we've often talked to the child's parents and then we will either set up that consultation, or the pediatrician will set it up if he wishes and that's the pediatrician's choice basically, do you want to call, do you want us to call. Even if the pediatrician does call, we will call our center and alert them to the fact that you're going to get this call from Dr. So and So about baby so and so, it's a presumptive positive for MSUD, we're faxing you the results. (Mid-Atlantic NBS Program)

We monitor turn around time for each of the individual disorders that we're screening for, in other words we'll have a turn around time for hemoglobin screening, we provide a turn around time for hyperthyroid screening, but we do each of the disorders individually, and then we monitor our time for the reporting of results. On an annual basis, we do a summary of time to treatment for each of the disorders, and then we do an evaluation of the hospitals on the adequacy of their initial screening. We monitor every child that's born through an independent reporting system, and match that against specimens 
that are received in our facility. We get the reports back on how they've done that screening, for all their kids, and whether or not they've met the funding requirements for screening, met the specimen quality perimeters, and their turn around times for getting specimens mailed in and that sort of stuff. (Pacific Northwest NBS Program)

There was a level of comfort expressed by NBS programs when discussing their STFU activities that did not exist around LTFU. For example, the same type of activity (e.g., collecting data from providers on patient care) was talked about differently depending on whether the activity was directed toward diagnosis confirmation or long-term outcomes such as developmental progress of children with disorders. There was little hesitancy by any program when asked to describe what they did around STFU. In addition, a number of state NBS programs cast the STFU process, with its twin outcomes of gaining a confirmatory diagnosis and getting newborns into initial care as "detective work," and attaining successful outcomes in these two areas as leading to "closed cases." In this way, the programs constructed STFU activities in ways that had clearly defined beginning and ending boundaries, while also casting themselves in the role of champion in getting those cases closed.

The guiding principles of "tracking down the kids or family" and "managing the kids" care to diagnosis" were present in almost all discussions of STFU. The presentation of STFU as a bounded set of activities, with a clear blueprint for action, also coincided with the perception among all 38 NBS programs that their involvement in everything up to the point of confirmatory diagnosis was appropriate and necessary. They saw themselves as the lead entity in STFU, over and above any role played by physicians or other child health programs.

The babies have to be found right away, and the diagnosis clarified. That's our most important job after testing. We are the ones who do that, and we're the ones who should be doing that. (Southern NBS Program)

I call and make sure they have the first appointment with the specialist, and I follow them until they have either gotten a normal result, or we have a confirmation of the disorder. Once I've completed those things, I close the case. (Southern NBS Program)

We identify all abnormals and make sure they get followed through to diagnosis. We can do that, because the number of abnormals is usually a reasonable one, but all our energies go into that. We stay on top of the kids and their families, make sure they have appointments set up with their medical homes or a specialist for further testing, and we have to physically get the follow-up documentation in order to let it go. We're very strict about that. (Midwest NBS Program)

\section{Involvement in direct patient care and LTFU}

Besides their roles in STFU, a quarter of the 38 state programs also saw themselves as having a role in providing direct services to patients over time. This cultural aspect undermined their ability to see LTFU in more expansive, population-based ways. The states seeing themselves this way tended to have one of the following: (a) one or more NBS program staff which had clinical roles working with care providers, or (b) clinical staff working in a specialty center that the NBS program funded directly. For example, one mid-Atlantic NBS program employed two nutritionists who had close contact with metabolic patients:

They are the ones who kind of keep tabs and have the personal relationship with the families over the long term. They help monitor care for individual patients, and participate in some of the specialty consults. (Mid-Atlantic NBS Program)

In another Northeast NBS program, the NBS coordinator is also a nurse who participates in the state's CSHN clinic, where most children with metabolic disorders are seen. The NBS program has a robust relationship with the clinic, where patients are seen by a multidisciplinary team, the family, and the primary care doctor. Each of these relationships is strengthened by a certain level of reciprocity. For instance, before a child is due for a clinic visit, the NBS program sends a letter to the primary care physician notifying him or her of the upcoming appointment and inviting the physician to write back with any updates on the child's condition and any particular concern. After the clinic visit, the NBS program then sends the primary care physician and family a copy of the notes from the clinic visit.

In these situations where more direct care relationships existed between the NBS program, providers, and families, there was little indication of in-depth, consistent LTFU data collection or use. In these state programs, data tended to be stored in hard copy, and staff reported not using these data for any type of long-term analysis. In one Southern NBS program, a nursecoordinator is assigned to follow each category of disorder and to address any barriers patients have in accessing care. Each nurse-coordinator keeps notes relevant to long-term follow up activities, and the state NBS program receives an annual report that includes the child's immunization status; whether they have been referred or enrolled in EI services; any change in diagnosis or insurance status; whether they have received genetic counseling; and the number of visits to the pediatric subspecialist. But when asked, this NBS program stated that the data were not used for monitoring patient care provided at a group or population level, evaluating specialty center performance, or tracking developmental or treatment-related outcomes of patients with specific disorders.

State programs collecting data past the confirmatory diagnosis phase that also involved themselves in direct patient care projected a collective belief that it was as important to be involved in providing direct services for individual patients as collecting data to evaluate or monitor anything at group or population levels of analysis. Any LFTU activities were directed solely at individual-specific progress, and less rooted in a public health orientation that considered what data could be collected and reported on patient populations over time, whether or not actual patient encounters occurred. LTFU data collection and usage in state NBS programs that had direct patient care involvement was reported 
as more sporadic and dependent upon whether or not a patient visit occurred.

\section{DISCUSSION}

Key cultural norms found in this study among NBS programs that shape beliefs and activities related to LTFU include (a) strong beliefs in STFU as the central aspect of their program's role, (b) an orientation toward LTFU that focuses on direct patient care and less on population-based activities like surveillance, and (c) perceptions that "someone else" is either doing LTFU within the particular state or better suited to doing it than the NBS program. Several observations can be drawn from the findings. First, they support the importance of understanding state NBS program culture and how that culture may shape the scope and substance of LTFU in a given state. Although the availability of staff and resources to conduct LTFU activities are key factors in whether or not NBS programs engage meaningfully in LTFU, this is the first known study to highlight how the beliefs and experiences of NBS programs may be linked to the conduct of LTFU. The importance of culture in this regard cannot be understated, especially because these programs have always been preoccupied with conducting testing and STFU in high quality ways. They are also now being asked to take on greatly expanded responsibilities in these same two areas.

Several of the findings hint at historical reasons why NBS programs think and act in the manner they do around LTFU. For example, in some state programs which have evolved with staff that is primarily from provider backgrounds (e.g., nursing), it is less surprising that one might find cultures oriented more to direct patient care, or to the notion that clinical specialists are better able to conduct LTFU than state NBS programs. In this way, reorienting NBS program cultures to become more accepting and engaged in population-based public health definitions of LTFU will involve staffing NBS programs in a manner that produces beliefs and actions supporting this definition. For instance, this might include more staff that has formal public health training and skills in areas such as epidemiology and biostatistics.

A second observation from the study findings is that the twin emphases on: (a) making sure that STFU gets done and (b) viewing direct care providers as "the experts" can undermine NBS programs from being proactive with respect to LTFU activities such as surveillance and quality assurance. If NBS programs believe that they are not suited to doing LTFU, or that in doing LTFU they come into conflict with the patient care system which treats diagnosed patients, it may matter less what resources are provided to these programs for doing LTFU. Resources are always an important factor, as staff in this study believed, but it is equally important to change program mindsets. This may involve, among other things, development of national standards for LTFU that all NBS programs use as guides, increased dialogue among state NBS programs to share best practices, and legislative actions that clearly demarcate the responsibilities of the state in relation to LTFU activities.
This study is limited in key ways. First, not all state NBS programs participated in the interviews. However, as noted the states not participating did not differ from participating states in terms of program-related variables. Second, the data are qualitative and subject to the limitations of all qualitative research. These limitations include a lack of generalizability and subjective bias in interpreting data. However, a justification for using qualitative methods was to gain deeper insight into a less understood topic, i.e., the cultures of state NBS programs. Gaining this rich description is not easily done through quantitative methods. The systematic approach taken to analyzing the data protects against these limitations. Finally, the sampling frame consisted of NBS follow-up coordinators and additional staff with knowledge of LTFU. Follow-up coordinators may not be the only source of cultural data on NBS programs. Yet, they arguably represent core staff that is required to think about and do LTFU. Their attitudes, beliefs, and behaviors are central to NBS follow-up culture.

In conclusion, the findings here are consistent with those reported quantitatively in other published studies of the NBS program role in $\mathrm{LTFU}^{3,5,11}$ in that state NBS programs seem to have significant handicaps presently in their ability to play a central role in the LTFU part of the NBS system. The question emerging from this accumulating body of research is whether or not state NBS programs can evolve enough in the near future to contribute significantly to the growing national emphasis on conducting LTFU. It is an important question that merits continued study. But taken as a whole, the results are not encouraging. In particular, this study highlights the importance of focusing on and changing NBS program culture to improve LTFU within a state, moving it more toward the kinds of population-based activities that define a public health orientation.

\section{ACKNOWLEDGMENTS}

The research reported in this article was part of a larger study supported through contract \#120807/120805 with the National Newborn Screening and Genetics Resource Center (NNSGRC) at the University of Texas Health Science Center at San Antonio.

The author was the Principal Investigator of the study and had full access to all the data in the study and takes responsibility for the integrity of the data and the accuracy of the data analysis. Special thanks go to Bradford Therrell, Director of the NNSGRC, and Maria Ayoob, for their assistance in various stages of the research.

\section{References}

1. Newborn Screening Task Force, American Academy of Pediatrics. Serving the family from birth to the medical home-Newborn screening: a blueprint for the future. Pediatrics 2000;106(suppl):383-427.

2. Maternal and Child Health Bureau. Newborn screening: toward a uniform screening panel and system. Washington DC: MCHB, Health Resources and Services Administration, 2004. Available at: ftp://ftp.hrsa.gov/mchb/genetics/screeningdraftforcomment. pdf. Accessed online on January 3, 2008.

3. Hoff T, Hoyt A. Practices and perceptions of long-term follow-up among state newborn screening programs. Pediatrics 2006;117:1922-1929.

4. Clayton EW. Issues in state newborn screening programs. Pediatrics 1992;90:641-646. 
5. Hoff T, Ayoob M, Therrell B. Long term follow-up data collection and use in state newborn screening programs. Arch Pediatr Adolesc Med 2007;161:994-1000.

6. Howell RR, Engelson G. Structures for clinical follow-up: newborn screening. J Inherit Metab Dis 2007;30:600 -605.

7. McCabe LL, Therrell BL Jr, McCabe ERB. Newborn screening: rationale for a comprehensive, fully integrated public health system. Mol Genet Metab 2002;77:267-273.

8. Clinical and Laboratory Standards Institute. Newborn screening follow-up: Approved guideline. CLSI document I/LA-27A. Wayne, PA: Clinical and Laboratory Standards Institute, 2006.

9. Saxena A. Issues in newborn screening. Genet Test 2003;7:131-134.
10. Advisory Committee on Heritable Disorders and Genetic Diseases in Newborns and Children. Meeting Minutes of Sixth Meeting, October 20-21, 2006. Accessed at: http://mchb.hrsa.gov/programs/genetics/committee/sixthmtgmins.htm. Accessed September 28, 2007.

11. Hoff T, Hoyt A, Therrell B, Ayoob M. Exploring barriers to long-term follow-up in state newborn screening programs. Genet Med 2006;8:563-570.

12. Strauss A. Qualitative analysis for social scientists. New York: Cambridge University Press, 1987.

13. Miles MB, Huberman AM. Qualitative data analysis: an expanded sourcebook, 2nd ed. Thousand Oaks, CA: Sage Publications, 1994. 